\title{
Resonant Raman scattering study of InSb etched by reactive ion beam etching
}

\author{
J. R. Sendra, ${ }^{\text {a) }}$ G. Armelles, T. Utzmeier, J. Anguita, and F. Briones \\ Instituto de Microelectrónica de Madrid, C.N.M., C.S.I.C., C/ Serrano 144, Madrid 28006, Spain
}

(Received 2 January 1996; accepted for publication 21 February 1996)

\begin{abstract}
A Raman study of InSb etched by reactive ion beam etching using a $\mathrm{CH}_{4} / \mathrm{H}_{2} / \mathrm{N}_{2}$ plasma generated by electron cyclotron resonance is presented. The evolution of the LO, 2LO phonon and phononplasmon coupled modes has been studied using resonant Raman spectra in different configurations. Results indicate an increase of the carrier density by a factor of about 60 and a decrease of the built-in potential due to the plasma process. The combination of both evolutions results in a prevalence of the electric field induced scattering upon the defect induced scattering mechanism. (C) 1996 American Institute of Physics. [S0021-8979(96)01111-2]
\end{abstract}

Long-wavelength optical devices fabricated on InSbbased III-V compound semiconductors are promising as infrared diodes. Wavelengths greater than $12 \mu \mathrm{m}$ can be detected with these compound based photodiodes at $77 \mathrm{~K}$. However there are few studies on the dry etching technology of these materials. They are based on different gas plasmas such as chlorine, ${ }^{1}$ iodine, ${ }^{2,3}$ freon $^{4}$ and hydrocarbons. ${ }^{4,5}$ Plasma etching technology introduces damage that can affect the performance of the devices and therefore there is a need to evaluate it. Although there are studies about the damage caused by ion bombardment in an InSb surface cleaning process,${ }^{6}$ no study about process-induced optical modifications in InSb has been found by the authors.

In this work the modification of the surface electric field, density of charge defects and lattice disruption induced by the etching process is studied by resonant Raman scattering. ${ }^{7}$ The evolution of those magnitudes can be obtained from the Raman spectra in four different backscattering configurations: $\bar{z}(x x) z, \bar{z}\left(x^{\prime} x^{\prime}\right) z, \bar{z}\left(y^{\prime} y^{\prime}\right) z$ and $\bar{z}(x y) z$, in this notation $\mathrm{x}, \mathrm{y}, \mathrm{z}, x^{\prime}$ and $y^{\prime}$ denote [100], [010], [001], [110] and $[1 \overline{1} 0]$ respectively.

The intensities of the longitudinal optical (LO) phonon peak in the four configurations can be expressed as: ${ }^{8}$

$$
\begin{aligned}
& \mathrm{LO}_{\bar{z}(x x) z}=\int_{0}^{L_{S}}\left(\left|a_{f}\right|^{2}+\left|a_{I}\right|^{2}\right) \exp (-\alpha z) d z, \\
& \mathrm{LO}_{\bar{z}\left(x^{\prime} x^{\prime}\right) z}=\int_{0}^{L_{S}}\left(\left|a_{f}+a_{\mathrm{dp}}\right|^{2} / 2+\left|a_{I}\right|^{2}\right) \exp (-\alpha z) d z, \\
& \mathrm{LO}_{\bar{z}\left(y^{\prime} y^{\prime}\right) z}=\int_{0}^{L_{S}}\left(\left|a_{f}-a_{\mathrm{dp}}\right|^{2} / 2+\left|a_{I}\right|^{2}\right) \exp (-\alpha z) d z, \\
& \mathrm{LO}_{\bar{z}(x y) z}=\int_{0}^{L_{S}}\left|a_{\mathrm{dp}}\right|^{2} \exp (-\alpha z) d z,
\end{aligned}
$$

where $\alpha$ is the absorption coefficient, $L_{S}$ the width of the surface deplexion region (SDR) and $a_{f}, a_{\mathrm{dp}}$ and $a_{I}$ are the Raman tensor elements for dipole forbidden scattering, dipole allowed scattering and impurity or defect-induced scat-

${ }^{a)}$ Electronic mail:Jose@imm.cnm.csic.es tering respectively. The dipole forbidden scattering is in fact composed of two terms, one intrinsic to and the other proportional to the electric field.

InSb residual doped $n$-type $\left(10^{17} \mathrm{~cm}^{-3}\right)$ layers were grown on InP (100) substrates in a conventional molecular beam epitaxy chamber with solid effusion cells. They consist of a $1000 \AA$ buffer layer grown at $380^{\circ}$ by molecular beam epitaxy followed by a $1.5 \mu \mathrm{m}$ thick layer grown at $300{ }^{\circ} \mathrm{C}$ by atomic layer molecular beam epitaxy.

A commercial load-lock electron cyclotron resonance reactive ion beam etching (RIBE) system was used for the plasma etching process. The electron cyclotron resonance plasma is generated by $2.45 \mathrm{GHz}$ microwave excitation and the magnetic field is driven by an electromagnet. A dc ion beam voltage was applied between the extraction grid and the grounded sample holder. The microwave power was set at $300 \mathrm{~W}$. Ion beam voltage was varied between $100 \mathrm{~V}$ and $500 \mathrm{~V}$. All the etchings were performed at room temperature for $15 \mathrm{~min}$. Details about the system can be found elsewhere. ${ }^{9}$ A flow of $14 \mathrm{sccm}$ of $\mathrm{CH}_{4} / \mathrm{H}_{2}\left(15 \% \mathrm{CH}_{4}\right)$ and 6 sccm of $\mathrm{N}_{2}$ was used due to the good morphology of the resulting samples and the inexistence of polymer deposition. Final surfaces that reproduce the morphology of the unetched ones were obtained.

The Raman spectra were obtained at $80 \mathrm{~K}$ using a triple spectrometer incorporating a charged coupled device camera system. The $647.1 \mathrm{~nm}$ line of a Krypton laser was used, then the energy excitation was $1.92 \mathrm{eV}$, close to the $1.96 \mathrm{eV}$ measured for the $E_{1}$ transition of $\mathrm{InSb}$ at $80 \mathrm{~K}$. The probed zone with this excitation will be about $190 \AA$.

Figure 1 shows the average etch rate of InSb as a function of the ion beam voltage. This figure reproduces previous results obtained when etching InP using the same technology. ${ }^{9}$ A threshold voltage $\left(V_{\text {th }}\right)$ of about $130 \mathrm{~V}$ followed by a constant slope line at higher ion beam voltages is observed. Etch rate values are slightly lower than the InP. These similarities can be explained by the behavior of the hydrides of $\mathrm{P}$ and $\mathrm{Sb}$, the expected reaction products. Both of them have boiling points below room temperature, $-88^{\circ} \mathrm{C}$ and $-17{ }^{\circ} \mathrm{C}$ respectively, ${ }^{10}$ thus indicating that the $\mathrm{PH}_{3}$ volatility can be higher than the $\mathrm{SbH}_{3}$ one, with a slight increase of the etch rate of InP with respect to InSb.

The information from the four configurations in the reso- 


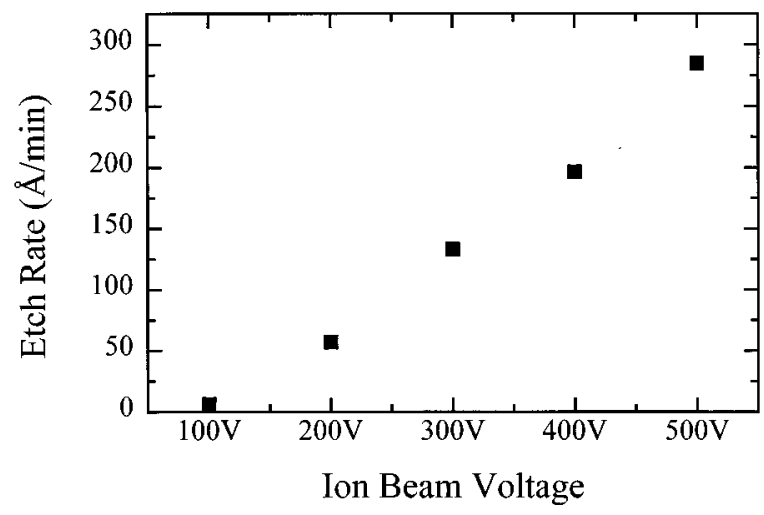

FIG. 1. Average etch rate of InSb over a 15 min period as a function of the ion beam voltage.

nant Raman spectra will now be extracted from three different features. The first feature is the LO phonon peak, the next one is the $2 \mathrm{LO}$ phonon peak and the last one is the plasmonphonon coupling mode, $L_{-}$peak, (the $L_{+}$plasmon mode is strongly damped and hardly observed). ${ }^{11}$ The volume that contributes to the $\mathrm{LO}$ or $2 \mathrm{LO}$ phonon scattering is located in the SDR while the $L_{-}$mode arises from the non-depleted volume. Then the intensity of every peak will depend on the scattering cross sections and of course on the probed volume. To neglect the latter contribution we have normalized every LO peak intensity with respect to the intensity of the LO phonon peak taken in the $\bar{z}(x y) z$ configuration, provided that the scattering intensity of the dipole allowed scattering $(\bar{z}(x y) z$ configuration) does not depend on the etching conditions. This possible dependence can be detected by measuring the ratio between the intensity of the $2 \mathrm{LO}$ phonon peak, taken from any spectra where the incident and scattered light are parallel, and the LO phonon peak taken in the $\bar{z}(x y) z$ configuration. This ratio is very sensitive to the lattice damage. ${ }^{12}$ The evolution of this ratio confirms the former assumption about the unmodified dipole allowed scattering intensities.

Raman spectra are modified as we increase the ion beam voltage. Remarkable differences appear in the spectra (see Figure 2). A new feature appears on the low energy side of

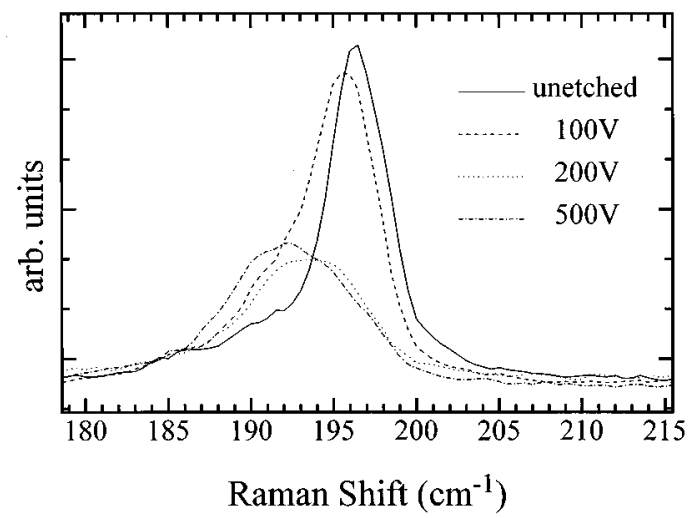

FIG. 2. Raman spectra of the unetched, $100 \mathrm{~V}, 200 \mathrm{~V}$ and $500 \mathrm{~V}$ etched samples taken in the $\bar{z}(x y) z$ configuration.

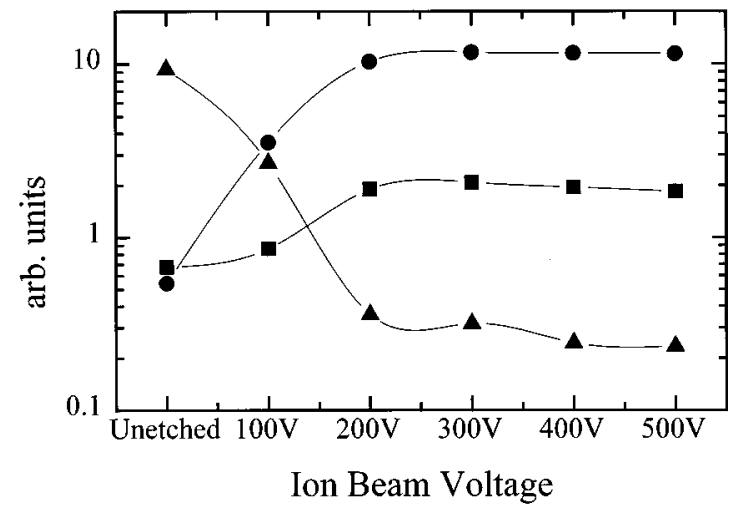

FIG. 3. Ratio as a function of the ion beam voltage of a) $\mathrm{LO}_{\bar{z}(x y) z} / L_{-}$ (squared symbols) b) $\left(\mathrm{LO}_{\bar{z}\left(x^{\prime} x^{\prime}\right) z}-\mathrm{LO}_{\bar{z}\left(y^{\prime} y^{\prime}\right) z}\right) / \mathrm{LO}_{\bar{z}(x y) z}$ (circled symbols) and c) $\mathrm{LO}_{\bar{z}(x x) z} / \mathrm{LO}_{\bar{z}(x y) z}$ (triangled symbols).

the LO Raman peak in the etched samples. This feature becomes dominant for ion beam voltages greater than $200 \mathrm{~V}$. This feature is hardly observed in the $\bar{z}(x x) z$ spectra and its intensity is lower than the LO peak for the other two configurations, $\bar{z}\left(x^{\prime} x^{\prime}\right) z$ and $\bar{z}\left(y^{\prime} y^{\prime}\right) z$, therefore we associate this feature with a plasmon-like peak $\left(L_{-}\right){ }^{13}$

As the etching conditions do not modify the dipole allowed scattering intensities, the SDR evolution with the ion beam voltage can be calculated from the relation between the intensities of the plasmon-like peak and the LO phonon peak, both taken in the $\bar{z}(x y) z$ configuration. ${ }^{14}$ Equation (5) shows this relation

$$
\frac{\mathrm{LO}_{\bar{z}(x y) z} / L_{-}}{\mathrm{LO}_{\bar{z}(x y) z}^{0} / L_{-}^{0}}=\frac{\exp \left(2 L_{S} / d\right)-1}{\exp \left(2 L_{S}^{0} / d\right)-1}
$$

where $L_{-}$is the intensity of the plasmon-phonon coupled mode, LO the intensity of the LO phonon peak, $L_{S}$ is the SDR width, $d$ is the probing depth and the superscript 0 means unetched sample. The relationship between the plasmon-like $L_{-}$and the dipole allowed LO intensities are shown in figure 3. A drastic reduction of the SDR width can be deduced from this evolution. For the sample etched with an ion beam voltage of $500 \mathrm{~V}$ the SDR width is about $5 \%$ of the unetched sample value.

The reduction of the SDR width can be due to the reduction of the built-in potential $\left(V_{\mathrm{bi}}\right)$ and/or to an increase of the carrier concentration (a constant value of the carrier concentration will be assumed inside the probed volume). The relation between the surface electric field and the $V_{\mathrm{bi}}$ is given as $E_{S}=V_{\mathrm{bi}} / L_{S}$, therefore the evolution of $V_{\mathrm{bi}}$ can be obtained from the evolution of $E_{S}$ and $L_{S}$. The evolution of $L_{S}$ was obtained in the preceding paragraph and the evolution of $E_{S}$ can be obtained from the following ratio: 


$$
\begin{array}{r}
\frac{\mathrm{LO}_{\bar{z}\left(x^{\prime} x^{\prime}\right) z}-\mathrm{LO}_{\bar{z}\left(y^{\prime} y^{\prime}\right) z}}{\mathrm{LO}_{\bar{z}(x y) z}} \\
=\frac{\int_{0}^{L_{S}} 2 a_{f}(z) a_{\mathrm{dp}} \cos (\theta) \exp (-\alpha z) d z}{\int_{0}^{L_{S}} a_{\mathrm{dp}}^{2} \exp (-\alpha z) d z} \\
a_{f}(z) \approx a_{F} E_{S} \frac{\left(L_{S}-z\right)}{L_{S}},
\end{array}
$$

LO is the intensity of the LO phonon taken in the underscript configuration, $a_{f}$ and $a_{\mathrm{dp}}$ are the Raman tensors for dipole forbidden and dipole allowed scattering, $\alpha$ is the absorption coefficient, $\theta$ is the angle between the vectors whose modules are $a_{f}$ and $a_{\mathrm{dp}}$ and $a_{F}$ is the electric field-induced factor for the Raman tensor for dipole forbidden scattering. In the calculation we have taken advantage of the fact that the dipole allowed scattering is not affected by the etching conditions $\left(a_{\mathrm{dp}}\right.$ is constant), and the electric field induced scattering is the predominant term in the dipole forbidden scattering $^{8}\left(a_{f}(z) \propto E(z)\right)$.

A monothonic reduction of $V_{\mathrm{bi}}$ results, the value of the sample etched at $500 \mathrm{~V}$ is about $15 \%$ of the $V_{\mathrm{bi}}$ for the unetched sample. The values of the carrier concentration can be obtained assuming that $N_{d}=E_{S} L_{S}$. An increase in a factor of around 60 is obtained when the ion beam voltage is $500 \mathrm{~V}$.

The ratio between the $\mathrm{LO}_{\bar{z}(x x) z}$ and $\mathrm{LO}_{\bar{z}(x y) z}$ intensities is proportional to the sum of the dipole forbidden (intrinsic and electric-field induced) and impurity-induced scattering [Equation (7)],

$$
\frac{\int_{0}^{L_{S}}\left(\left|a_{f}\right|^{2}+\left|a_{I}\right|^{2}\right) \exp (-\alpha z) d z}{\int_{0}^{L_{S}} a_{\mathrm{dp}}^{2} \exp (-\alpha z) d z} .
$$

In non-doped samples the impurity induced scattering mechanism is greater than the dipole forbidden scattering. ${ }^{15}$ In doped samples, due to the increase of the surface electric field, it is not clear which is the predominant term (electric field induced or impurity induced mechanism). To determine which is the main term the $\mathrm{LO}_{\bar{z}(x x) z} / \mathrm{LO}_{\bar{z}(x y) z}$ ratio has been calculated as a sum of both components with $E_{S}$ obtained from equation 6 and $N_{d}$ from $E_{S}$ and the SDR. The result reveals the electric field induced scattering as the main term although the impurity-induced mechanism increases its weight when the ion beam voltage increases. The increasing value of the impurity induced scattering over the electric field-induced mechanism is due to the reduction of SDR as $E_{S}=N_{d} L_{S}$. Therefore, for this wavelength the impurityinduced scattering mechanism is lower than the electric field induced mechanism in these samples.

The carrier density increase can be attributed to the generation of $\mathrm{Sb}$ vacancies, it is well known that the hydrogenbased plasmas produce preferential etching of the $\mathrm{V}$ group element. In fact, preliminary Auger electron spectroscopy (AES) measurements detect a shallow region where the In to $\mathrm{Sb}$ ratio increases enough to be detected with this technique, then if the levels generated due to this lattice defect behave as donors they will contribute to the carrier density.

An optical characterization of the process-induced optical modification by reactive ion beam etching of InSb using a $\mathrm{CH}_{4} / \mathrm{H}_{2} / \mathrm{N}_{2}$ plasma generated by electron cyclotron resonance has been shown. The evolution of the LO, 2LO phonon and phonon-plasmon coupled mode have been studied using resonance Raman spectra in different configurations. The results indicate an increase of the carrier density by a factor of about 60 , a decrease of the built-in potential due to the plasma process, and the combination of both evolutions results in a prevalence of the electric field-induced scattering upon the defect-induced scattering mechanism.

${ }^{1}$ G. A. Vawter and J. R. Wendt, Appl. Phys. Lett. 58, 289 (1991).

${ }^{2}$ S. J. Pearton, U. K. Chakrabarti, W. S. Hobson, C. R. Abernathy, A. Katz, F. Ren, T. R. Fullowan, and A. P. Perley, J. Electrochem. Soc. 139, 1763 (1992).

${ }^{3}$ U. K. Chakrabarti, S. J. Pearton, A. Katz, W. S. Hobson, and C. R. Abernathy, J. Vac. Sci. Technol. B 10, 2378 (1992).

${ }^{4}$ S. J. Pearton, W. S. Hobson, and F. A. Baiocchi, J. Electrochem. Soc. 137, 1924 (1990).

${ }^{5}$ J. R. Sendra, J. Anguita, J. J. Pérez-Camacho, and F. Briones. Appl. Phys. Lett. 67, 3289 (1995).

${ }^{6}$ T. S. Jones, M. Q. Ding, N. V. Richardson, and C. F. Conville, Surf. Sci. 247, 1 (1991).

${ }^{7}$ W. Pletschen, J. Wagner, G. Kaufel, and K. Köhler, Appl. Phys. Lett. 59, 2299 (1991).

${ }^{8}$ J. Menéndez and M. Cardona, Phys. Rev. B 31, 3696 (1985).

${ }^{9}$ J. R. Sendra and J. Anguita, Jpn. J. Appl. Phys. 33, L390 (1994).

${ }^{10}$ Dry Etching for Microelectronics, edited by R. H. Burton, R. A. Gottscho, and G. Smolinsky (Elsevier, Amsterdam, 1984).

${ }^{11}$ Light Scattering in Solids, edited by M. Cardona and G. Güntherodt (Springer, Berlin, 1985).

${ }^{12}$ J. Wagner and Ch. Hoffman, Appl. Phys. Lett. 50, 682 (1987).

${ }^{13}$ W. Kauschke, N. Mestres, and M. Cardona, Phys. Rev. B 36, 7469 (1987).

${ }^{14}$ H. Shen, F. H. Pollack, and R. Snacks, Appl. Phys. Lett. 47, 891 (1985).

${ }^{15}$ J. Menéndez, L. Viña, M. Cardona, and E. Anastassakis, Phys. Rev. B 32, 3966 (1985). 